Original article

\title{
Posthemorrhagic hydrocephalus with decompensation of cerebrospinal fluid dynamics in newborns: new in treatment tactics
}

\author{
Oleg V. Volkodav ${ }^{1}$, Svetlana A. Zinchenko ${ }^{1}$, William A. Khachatryan ${ }^{2}$ \\ ${ }^{1}$ V.I. Vernadsky Crimean Federal University, Simferopol, Russia \\ ${ }^{2}$ Polenov Research Institute of Neurosurgery - Branch of Almazov National Medical Research Center, St. Petersburg, Russia
}

Received 16 January 2020, Revised 6 September 2020, Accepted 5 November 2020

(C) 2020, Volkodav O.V., Zinchenko S.A., Khachatryan W.A.

(C) 2020, Russian Open Medical Journal

Abstract: Background - Posthemorrhagic hydrocephalus in newborns with occlusion of cerebrospinal fluid leads to decompensation of cerebrospinal fluid dynamics. There is no single method that meets all the criteria for the effectiveness and safety of treatment.

The study goal was to investigate the use of coronary translambdoid subarachnoid ventriculostomy (CTSV) and ventricular subarachnoid stenting (VSS) in the treatment of neonatal hydrocephalus.

Material and Methods - The analysis of the posthemorrhagic hydrocephalus treatment in 327 newborns for the period of 2000-2018 in Crimea. Two groups have been identified. In the Group 1, 184 children underwent standard treatment according to the 'LVV protocol' with lumbar and ventricular punctures with 20-22G needles, while with progression of hydrocephalus, with ventriculosubgaleal drainage and ventriculoperitoneal shunt. In 143 children with occlusion and ventricular block, the treatment complex included CTSV - RF Patent No. 2715535, and ventricular drainage by the ventricular subarachnoid stenting (VSS) - RF Patent No. 2721455.

Results - An increase in the treatment radicality under CTSV is achieved through the use of the cerebral needles of a larger diameter (14G) and puncture access zones, elimination of occlusion, while under VSS, restoration of intracranial circulation and absorption of cerebrospinal fluid is ensured by prolonged sanitation with a saline solution of cerebrospinal fluid spaces. A positive outcome with compensation for hydrocephalus was achieved in $75.4 \%$ of cases versus $28.2 \%$ under the conventional protocol $(p<0.001)$. In other cases, the imbalance of production and absorption of cerebrospinal fluid remained, which required the integration of the VSS with the peritoneal segment of the shunt, without further replacement and reinstallation of the system.

Conclusion - Our results allow us to consider the effectiveness of CTSV and VSS inclusion in the contemporary algorithm for the treatment of decompensated posthemorrhagic hydrocephalus in newborns.

Keywords: newborn infants, hydrocephalus, neurosurgical treatment.

Cite as Volkodav OV, Zinchenko SA, Khachatryan WA. Posthemorrhagic hydrocephalus with decompensation of cerebrospinal fluid dynamics in newborns: New in treatment tactics. Russian Open Medical Journal 2020; 9: e0413.

Correspondence to Oleg V. Volkodav. E-mail: oleg_vlad.volk@mail.ru.

\section{Introduction}

At present, much attention is paid to the study of posthemorrhagic hydrocephalus in newborns [1-14]. It is noted that the formation of hydrocephalus is due to direct occlusion of the cerebrospinal fluid by blood clots, or dynamic occlusion by means of the subarachnoid space (SAS) compression, impaired absorption of cerebrospinal fluid (CSF) with indications, at the initial stage, for lumbar punctures (LP), ventricular punctures (VP) with 20-22G needles, as well as puncture drainage of the lateral ventricles [1-5]. If CSF dynamics disorders persist, ventriculosubgaleal drainage (VSGD) and ventriculoperitoneal shunt (VPS) are performed [2], according to the so-called 'LVV protocol' (i.e. LP-VSGD-VPS). The relevance of the problem is that there is no generally accepted opinion regarding the treatment of decompensated hydrocephalus [6-8] with the need to personalize treatment tactics aimed at restoring CSF dynamics [2], reducing the incidence of VPS and system dysfunction [9-14].
The study goal was to investigate the use of translambdoid coronary subarachnoid ventriculostomy (CTSV) and ventricular subarachnoid stenting (VSS) in the treatment of posthemorrhagic hydrocephalus in newborns.

\section{Material and Methods}

We analyzed the treatment of 327 newborns with posthemorrhagic hydrocephalus was carried out based on the materials of the Crimean Republican Clinical Hospital for Children and the Perinatal Center for the period of 2000-2018. Two groups were identified. In the Group 1, 184 children underwent treatment according to the LVV protocol with LP and VP with 20-22G needles at the initial stage, and with the progression of hydrocephalus, VSGD and VPS (Table 1).

In the Group 2, the treatment complex for 143 children with ventricular occlusion and SAS block at the initial stage included CTSV (RF Patent No. 2715535). With the progression of 
hydrocephalus, the treatment complex involved ventricular drainage in the SAS by the VSS system (RF Patent No. 2721455), which is reflected in Table 2 .

CTSV is performed by puncture with $14 \mathrm{G}$ needles at two points through the coronary and lambdoid sutures of the anterior and occipital horns of the lateral ventricles with unloading of their blood and cerebrospinal fluid and SAS decompression (Figure 1). Sanitation is performed by saline solution of the ventricles with arachnoid encephalic lysis when the needles are shoved into the SAS. Between the ventricles and SAS, drainage canals with collateral CSF outflow and elimination of occlusion are formed. The procedure is repeated three times with four-day intervals, alternating with sanitation of the craniospinal CSF LP pathways, until CSF dynamics stabilization with clinical restoration of the outflow and absorption of CSF, based on neuroimaging data. The advantages of the method include the simplicity of its technical implementation (a child in the incubator), the safety and effectiveness of the ventricle sanitation from blood clots with minimization of brain injury during hemotamponade, and a reduction in the sanitation time of the craniospinal CSF spaces.

Draining the ventricles into the SAS by the VSS system was implemented through ventricular drainage and perforation of the pump base (Figure 2).

To do this, after immersion of the ventricular drainage into the ventricle with control of the CSF flow, the pump is installed in the milling hole with a diameter of up to $10 \mathrm{~mm}$ with the straightening of the fixing cuff in the SAS and fixation by suturing along the trepanation edges. Additionally, there is a temporary CSF outflow from the pump through a fragment of the distal drainage into the subgaleal pocket (SP), which makes it possible to smooth out the drops of intracranial pressure (ICP) in the postoperative period with unloading and sanitation of the CSF pathways. The dome of the pump is punctured, and a saline solution is injected with control of its outflow into the SAS and ventricles. Reintroduction of saline solution through a pump with active sanitation of the SAS and ventricles, and passive excretion of CSF through the SP in combination with LP is conducted at days $3-5,7,10$ and 14 , as well as at the end of the week $3,4,5$ and 6 after the surgery.

Table 1. Scope of neurosurgical care in Group 1

\begin{tabular}{lcc}
\hline $\begin{array}{l}\text { Stages of hydrocephalus correction according to } \\
\text { the LVV protocol }\end{array}$ & $\begin{array}{c}\text { Number of } \\
\text { children }\end{array}$ & $\%$ \\
\hline LP and VP with 20-22G needles & 184 & 100 \\
Ventriculosubgaleal drainage (VSGD) & 151 & 82.1 \\
Ventriculoperitoneal shunt (VPS) & 132 & 71.7 \\
\hline
\end{tabular}

Table 2. Scope of neurosurgical care in Group 2 Stages of hydrocephalus correction with inclusion of CTSV and Number of \% VSS

CTSV (with $14 \mathrm{G}$ needles) in combination with LP

Ventricular drainage by VSS system in combination with VSGD children

100

Integration of VSS system with the peritoneal shunt segment

$\begin{array}{cc}143 & 100 \\ 94 & 65.7\end{array}$

Table 3. Comparative analysis of hydrocephalus compensation among the groups

\begin{tabular}{|c|c|c|c|}
\hline Hydrocephalus compensation & $\begin{array}{l}\text { Number } \\
\text { children }\end{array}$ & $f \%$ & $p^{*}$ \\
\hline After LP and VP (according to LVV protocol) & 33 & 17.9 & \\
\hline After CTSV inclusion (in combination with LP) & 49 & 34.3 & 0.046 \\
\hline After VSGD (according to LVV protocol) & 19 & 12.6 & \\
\hline After VSS inclusion (in combination with VSGD and LP) & 59 & 62.8 & $<0.001$ \\
\hline
\end{tabular}

* based on Fisher's exact test.
While maintaining the disproportion between the increasing age-related volume of CSF production and its absorption after the week 6, the VSS system was integrated with the peritoneal segment of the shunt at medium pressure through the distal drainage.

The data were processed using the STATISTICA 6.0 software (StatSoft Inc., USA). Fisher's exact test was used to compare the percentages in the two groups. Significant changes in indicators were considered those, for which the probability of the null hypothesis was: $p<0.05$.

\section{Results}

Compensation of hydrocephalus with stabilization of CSF dynamics at the initial stage of treatment, according to the LVV protocol, in Group 1 after LP was noted in 33 of 184 children, which made it possible to exclude drainage operations in $17.9 \%$ of cases (Table 3).

When CTSV was included into the hydrocephalus treatment complex in Group 2, hydrocephalus compensation was observed in 49 of 143 children, which made it possible to exclude drainage operations in $34.3 \%$ of cases $(p=0.046)$. A increase in the drainage output after inclusion of CTSV was ensured by effective unloading of the ventricles from blood clots and CSF, using $14 \mathrm{G}$ needles, flushing the ventricles and SAS with saline solution, eliminating occlusion and shortening the sanitation time of the craniospinal CSF pathways.

The inclusion of the VSS system in the complex of hydrocephalus treatment ensured the restoration of the CSF dynamics balance without VPS, which is shown in Figure 3 .

In Group 1, compensation of hydrocephalus after VSGD was observed in 19 of 151 children (12.6\%), whereas after VSS inclusion in Group 2 treatment, it was noted in 59 of 94 children, which made it possible to exclude VPS in $62.8 \%$ of cases $(p<0.001)$.

The cumulative positive outcome of hydrocephalus compensation without VPS, after staged hydrocephalus correction with CTSV and VSS inclusion in the treatment complex, was achieved in $75.4 \%$ of cases versus $28.2 \%$ under the conventional protocol $(p<0.001)$.

In other cases, the VSS system was integrated with the VPS segment. At the same time, sanitation of the ventricles and SAS was ensured with saline solution through the stent pump, which made it possible to exclude dysfunction of the ventricular segment and the pump (noted in $31 \%$ of cases in Group 1), and no hyperdrainage was noted (versus $18 \%$ in Group 1 ).

To improve the efficiency of the VSS system, a self-expanding VSS (patent application No. US 2018/0071501 A1) with an additional sealing cuff was proposed for installing the stent in the milling hole without suturing and eliminating the threat of liquorrhea.

\section{Discussion}

Treatment of posthemorrhagic hydrocephalus in newborns involves performing LP at the initial stage. At the same time, the removal of blood clots from the ventricular system is not ensured, long-term sanitation of the craniospinal CSF spaces is required, and a thrombus in the region of the third ventricle and other types of CSF occlusion constitute a contraindication to this procedure [15]. 

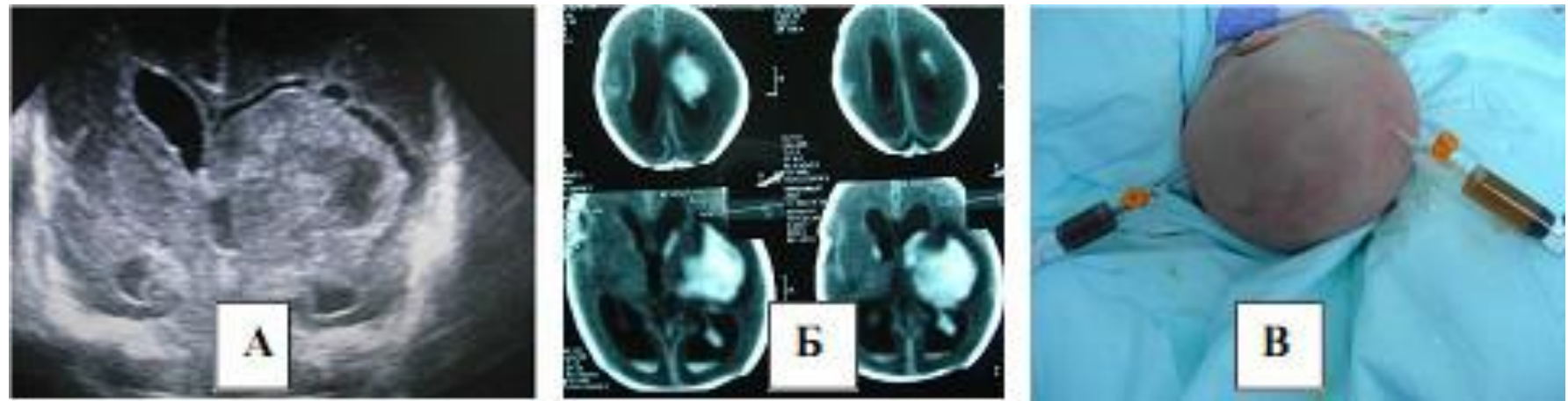

Figure 1. CTSV at the initial stage of hydrocephalus correction.
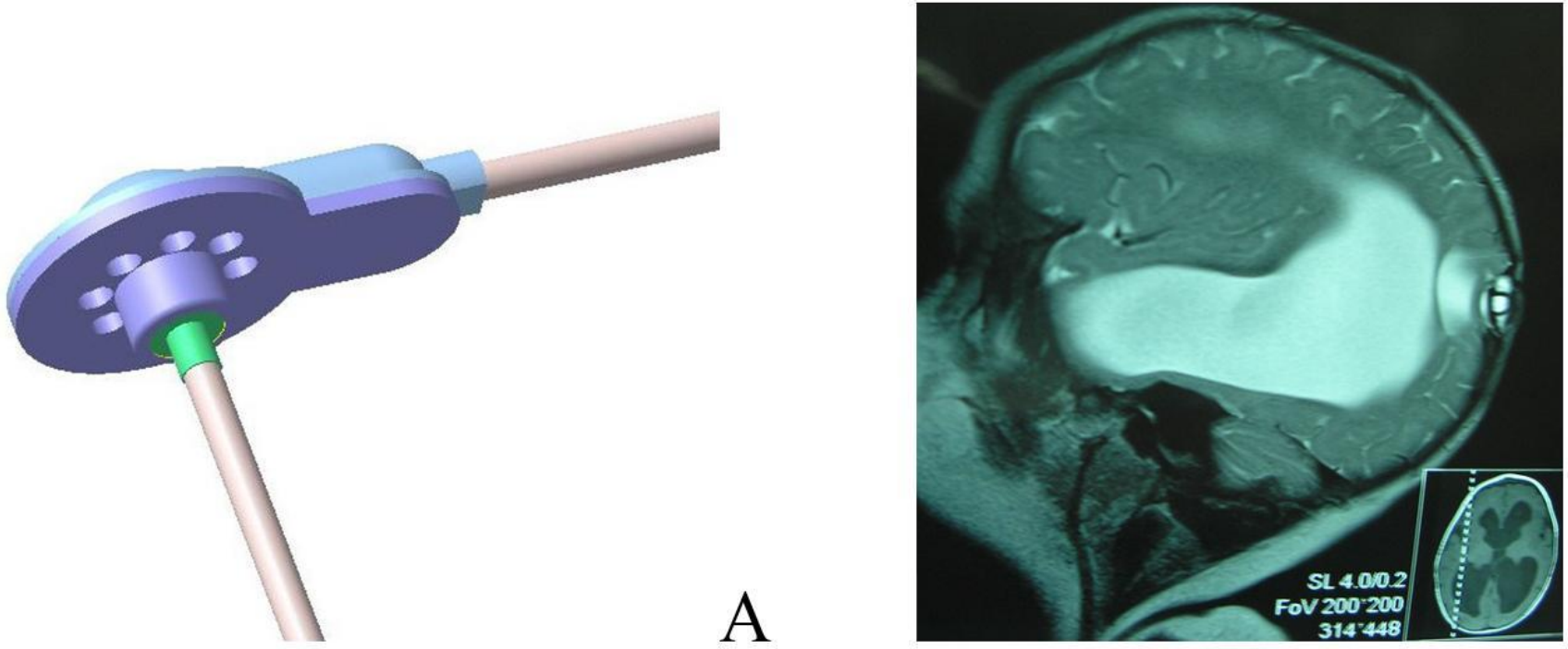

Figure 2. (A) VSS system, (B) MRI after the surgery.

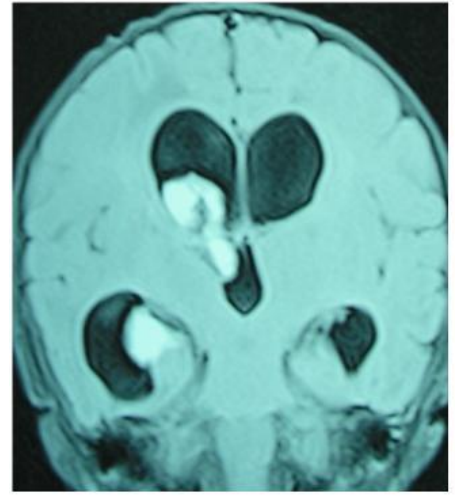

A

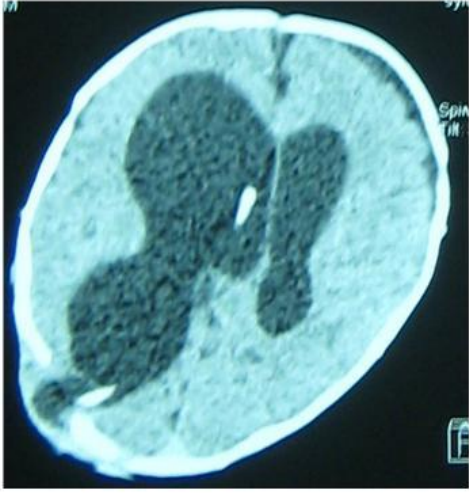

B

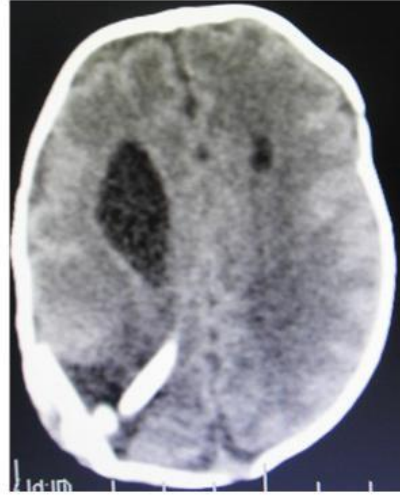

$\mathrm{C}$

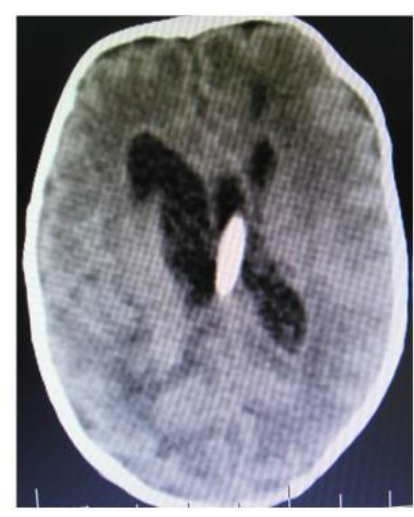

$\mathrm{D}$

Figure 3. Child S. with posthemorrhagic hydrocephalus, postconceptional age (PCA) 30 weeks, weight 1350 grams. (A) MRI at the initial stage. (B) Computed tomography (CT) after stenting with the VSS system at 38 weeks. (C-D) CT control 3 months after VSS.

VP needles of 20-22G type do not provide effective ventricular unloading from blood clots with a high risk of damage to the brain substance during their active aspiration, while under prolonged external puncture drainage, necessary for clot lysis, the risk of infection increases and the occlusion of the CSF is not eliminated [1-5].
An increase in the effectiveness of treatment via CTSV is achieved through the use of cerebral needles of larger diameter and puncture access zones with elimination of occlusion, sanitation of the ventricles from blood clots, and craniospinal SAS from the products of its decay, with a decrease both in the sanitation time and the risk of adhesions. 
VSGD provide long-term sanitation and unloading of the ventricles from blood and CSF without the elimination of dynamic CSF occlusion [1-3, 5].

An increase in the treatment effectiveness under VSS is achieved by restoring intracranial circulation and CSF absorption at the stage of prolonged sanitation of craniospinal CSF spaces with saline solution in combination with VSGD and LP. There is a high percentage of dysfunction and complications after VPS [6-9, 1214].

Integration of the VSS with the peritoneal segment of the shunt has provided CSF diversion into the SAS and dosed discharge of excess CSF into the abdominal cavity with adaptation of the resorptive capacity to the increasing volume of CSF production in the first year, and has reduced the risk of system dysfunction without its replacement and reinstallation.

\section{Conclusion}

The staged inclusion of CTSV and VSS into the treatment algorithm for decompensation of posthemorrhagic hydrocephalus in newborns, in combination with LP and VSGD, made it possible to increase the percentage of hydrocephalus compensation without VPS to $75.4 \%$ versus $28.2 \%$ under the conventional protocol $(p<0.001)$ by restoring intracranial circulation and CSF absorption. While maintaining disorders of CSF dynamics, the integration of the VSS system with the peritoneal segment of the shunt permitted to eliminate the imbalance in production and absorption of CSF with adaptation of the SAS resorptive capacity to increasing volume of CSF production in the first year, and to reduce the risk of dysfunction.

\section{Conflicts of Interest}

The authors declare no conflicts of interest.

\section{Ethical approval}

All procedures in our study involving human participants were performed in accordance with the ethical standards of the institutional research committee, as well as with the 1964 Declaration of Helsinki and its later amendments or comparable ethical standards.

\section{References}

1. Intraventricular hemorrhage, posthemorrhagic hydrocephalus in newborns, principles of medical care. Guidelines. Volodin NN, Gorelyshev SK, Popov VE, eds. Moscow, Russia, 2014, 43 p. Russian. http://www.raspm.ru/files/VGK\%20u\%20novorogdennyh.pdf.

2. Kryukov EYu, lova AS, Andrushenko NV. Kryukova IA, Usenko IN. Personalization of posthemorrhagic hydrocephalus treatment in newborns. Neirokhirurgiya i Nevrologiya Detskogo Vozrasta 2017; (3(53)): $\quad 58-62 . \quad$ Russian. https://www.elibrary.ru/item.asp?id=32502232.

3. Zinenko DYu, Vladimirov MYu. A new approach to diagnosis and treatment of posthemorrhagic hydrocephalus in premature infants. Voprosy Prakticheskoi Pediatrii 2008; 3(3): 5-10. Russian. https://www.elibrary.ru/item.asp?id=11516208.

4. Whitelaw A, Lee-Kelland R. Repeated lumbar or ventricular punctures in newborns with intraventricular haemorrhage. Cochrane Database $\begin{array}{llll}\text { Syst Rev 2017; } & \text { 4(4): } & \text { CD000216. }\end{array}$ https://doi.org/10.1002/14651858.cd000216.pub2.

5. Zaben $M$, Finnigan $A$, Bhatti $\mathrm{MI}$, Leach $\mathrm{P}$. The initial neurosurgical interventions for the treatment of posthaemorrhagic hydrocephalus in preterm infants: A focused review. Br J Neurosurg 2016; 30(1): 7-10. https://doi.org/10.3109/02688697.2015.1096911.
6. Wellons JC 3rd, Shannon CN, Holubkov R, Riva-Cambrin J, Kulkarni AV, Limbrick DD Jr, et al. Hydrocephalus Clinical Research Network. Shunting outcomes in posthemorrhagic hydrocephalus: results of a Hydrocephalus Clinical Research Network prospective cohort study. J Neurosurg Pediatr 2017; 20(1): 1929. https://doi.org/10.3171/2017.1.peds16496.

7. Kulkarni AV, Sgouros $S$, Leitner $Y$, Constantini S; International Infant Hydrocephalus Study Investigators. International Infant Hydrocephalus Study (IIHS): 5-year health outcome results of a prospective, multicenter comparison of endoscopic third ventriculostomy (ETV) and shunt for infant hydrocephalus. Childs Nerv Syst 2018; 34(12): 23912397. https://doi.org/10.1007/s00381-018-3896-5.

8. Khachatryan WA, Samochernyh KA, Kim AV, Nikolaenko MS, Sysoev $\mathrm{KV}$, Don OA, et al. Ventriculo-sinus transversal shunt in the treatment of decompensated hydrocephalus in children (the results of clinical testing of the method) Translational Medicine 2017; 4(1): 20-28. Russian. https://doi.org/10.18705/2311-4495-2017-4-1-20-28.

9. Robinson S. Neonatal posthemorrhagic hydrocephalus from prematurity: pathophysiology and current treatment concepts. $J$ Neurosurg Pediatr 2012; 9(3): 242-258. https://doi.org/10.3171/2011.12.peds11136.

10. Tan AP, Svrckova P, Cowan F, Chong WK, Mankad K. Intracranial hemorrhage in neonates: a review of etiologies, patterns and predicted clinical outcomes. Eur J Paediatr Neurol 2018; 22(4): 690717. https://doi.org/10.1016/j.ejpn.2018.04.008.

11. Melo JRT, Passos RK, Carvalho MLCM. Cerebrospinal fluid drainage options for posthemorrhagic hydrocephalus in premature neonates. Arq Neuropsiquiatr 2017; 75(7): 433-438. https://doi.org/10.1590/0004-282×20170060.

12. Christian EA, Melamed EF, Peck E, Krieger MD, McComb JG. Surgical management of hydrocephalus secondary to intraventricular hemorrhage in the preterm infant. I Neurosurg Pediatr 2016; 17(3): 278-284. https://doi.org/10.3171/2015.6.peds15132.

13. Kim HM, Kim KH. Clinical experience of infantile posthemorrhagic hydrocephalus treated with ventriculo-peritoneal shunt. Korean $J$ Neurotrauma 2015; 11(2): 106-111. https://doi.org/10.13004/kjnt.2015.11.2.106.

14. Di Rocco C, Turgut M. Jallo G, Martínez-Lage DJF, eds. Complications of CSF shunting in hydrocephalus: prevention, identification, and management. Springer International Publishing, 2015; 322 p. https://doi.org/10.1007/978-3-319-09961-3.

\section{Authors:}

Oleg V. Volkodav - PhD, Associate Professor, V.I. Vernadsky Crimean Federal University, Simferopol, Russia. https://orcid.org/0000-0001-96625731.

Svetlana A. Zinchenko - PhD, Associate Professor, V.I. Vernadsky Crimean Federal University, Simferopol, Russia. https://orcid.org/0000-0002-5222$\underline{2110}$.

William A. Khachatryan - MD, Professor, Polenov Research Institute of Neurosurgery - Branch of Almazov National Medical Research Center, St. Petersburg, Russia. https://orcid.org/0000-0002-1635-6621 